\title{
An electron microscopy study of $\beta$-FeOOH (akaganéite) nanorods and nanotubes
}

\author{
William R. Richmond, ${ }^{* a}$ John M. Cowley, ${ }^{b}$ Gordon M. Parkinson ${ }^{a}$ and Martin Saunders ${ }^{c}$ \\ Received 21st September 2005, Accepted 29th November 2005 \\ First published as an Advance Article on the web 13th December 2005 \\ DOI: $10.1039 / b 513423 d$
}

High-resolution TEM images reveal that samples of $\beta$-FeOOH (akaganéite) prepared by quenching of a condensed ferrihydrite gel contain a mixture of rod-like particles and tubes. The tubular particles are usually about $10-15 \mathrm{~nm}$ in diameter with a central void that is typically $1 / 3$ of the particle diameter. Dark-field STEM images show that the tubular particles are made up of single crystals that extend across the whole tube diameter, but only rarely extend along the whole length of the tube. Both the solid rods and the tubes appear to be based on subunits of approximately $3-4 \mathrm{~nm}$ in diameter, and it is proposed that formation of the akaganéite particles, both tubes and rods, results from secondary nucleation of these subunits at sites on particle edges, followed by rapid linear growth along the $c$-direction of the akaganéite structure.

\section{Introduction}

The iron oxyhydroxide $\beta$-FeOOH can be formed in either a somatoidal (cigar-shaped) morphology, or as rod-like particles of approximately 10-30 nm diameter. ${ }^{1}$ The morphology of this iron oxyhydroxide phase, also known by its mineral name, akaganéite, has been the subject of considerable interest and debate for many years. A study of somatoidal akaganéite published by Watson et al. ${ }^{2}$ in the 1960 s suggested that this morphology was comprised of "stacks" of hollow rods having pores of $3 \mathrm{~nm}$ average diameter. Subsequent work by Gallagher and Philips ${ }^{3,4}$ supported this conclusion on the basis of a reversible adsorption-desorption isotherm for nitrogen on somatoidal $\beta-\mathrm{FeOOH}$, which showed a maximum for pores with a diameter of $3 \mathrm{~nm}$. Such a system, with its regular porous substructure, was thought to be of considerable interest for adsorption studies, but later work investigating the porous structure of both somatoidal ${ }^{5,6}$ and rod-like ${ }^{7}$ akaganéite provided no evidence for $3 \mathrm{~nm}$ pores. The idea of akaganéite particles being composed of hollow rods was further refuted by the work of Galbraith et $a l^{8}{ }^{8}$ — an electron microscopy study of thin cross-sections of akaganéite particles that demonstrated the effects of beam-damage on the appearance of the sections. The hollow particles observed in earlier electron micrographs were thus attributed to artefacts arising from thermal decomposition of $\beta-\mathrm{FeOOH}$ in the electron beam. ${ }^{6,8}$

Somatoidal particles are generally synthesized by slow hydrolysis of acidic aqueous solution of iron(III) chloride, with reaction times of a number of days or even months. Crystals grown at room temperature display a square or stepped cross-section, but those grown at higher temperature tend to have a circular cross-section. ${ }^{1}$ The rod-like

${ }^{a}$ Nanochemistry Research Institute, Curtin University of Technology, GPO Box U1987, Perth, Western Australia, 6845, Australia

${ }^{b}$ Department of Physics and Astronomy, Arizona State University, Box 871504, Tempe, AZ 85287-1504, USA

${ }^{c}$ Centre for Microscopy and Microanalysis, The University of Western Australia, Crawley, Western Australia, 6009, Australia morphology occurs under conditions promoting much more rapid hydrolysis in $\mathrm{Fe}(\mathrm{III})$ solutions that are partly neutralized (i.e. $0<\mathrm{OH} / \mathrm{Fe}<3$ ). ${ }^{9}$ Akaganéite also arises as an intermediate phase in acid hydrolysis of condensed iron(III) hydroxide gels under conditions that are often employed in the preparation of uniform colloidal hematite particles. ${ }^{10} \mathrm{We}$ have isolated the intermediate akaganéite phase by rapid cooling of such systems to "quench" the phase transformation, obtaining nanoparticulate akaganéite samples comprised of rods $50-100 \mathrm{~nm}$ in length and $\sim 10 \mathrm{~nm}$ diameter. Examination of these particles by electron microscopy has indicated that some of the particles are hollow rods. This paper presents evidence for the existence of such hollow rods and describes some attempts to explain their formation.

\section{Experimental}

In a $100 \mathrm{ml}$ Nalgene screw-top plastic jar, $25 \mathrm{ml}$ of $2.0 \mathrm{~mol} \mathrm{~L}^{-1}$ iron(III) chloride solution was added to $28.5 \mathrm{ml}$ of $5.26 \mathrm{~mol} \mathrm{~L}^{-1}$ sodium hydroxide solution. The resulting iron oxyhydroxide suspension was mixed thoroughly by vigorous shaking of the jar. The $\mathrm{pH}$ was adjusted to 2 with a small amount of sodium hydroxide and/or hydrochloric acid and the suspension was aged at $98{ }^{\circ} \mathrm{C}$ for 4 hours in a laboratory oven. On removal from the oven the mixture was rapidly cooled in an ice bath. The solid was washed with water and ethanol by repeated settling/re-suspension cycles using a centrifuge, then collected and dried in air at $55{ }^{\circ} \mathrm{C}$ for two days.

For transmission electron microscopy (TEM), the samples were dispersed in ethanol with the aid of ultra-sonication, then cast and dried onto a conventional holey carbon-coated copper grid. Transmission electron micrographs were recorded on a JEOL 2011 TEM operating at $200 \mathrm{kV}$ and on a JEOL 3000 FEG TEM operating at $300 \mathrm{kV}$.

Scanning transmission electron microscopy (STEM), and electron nanodiffraction were performed using a Vacuum Generators HB-5 STEM that had been previously modified to allow convenient observation and recording of diffraction 
patterns or STEM images with various detector configurations. To obtain single-crystal diffraction patterns, a $100 \mathrm{keV}$ FEG electron beam with a cross-over diameter of $0.7 \mathrm{~nm}$ was focussed onto the sample. The method has been described in detail by Cowley and Spence, ${ }^{11}$ and the application of nanodiffraction to the characterization of iron oxides has also been extensively documented. ${ }^{12-14}$

\section{Results and discussion}

\section{HRTEM images}

Under the conditions of temperature and concentration employed in this work, hematite is the thermodynamically stable iron oxide phase, but akaganéite forms as an intermediate, and is the dominant phase in the condensed ferric hydroxide gel after a period of about 5 hours. The akaganéite particles formed in this reaction mixture are thin needles about $10 \mathrm{~nm}$ in diameter and vary in length from a few tens of nanometers to about $300 \mathrm{~nm}$. A TEM image of a typical sample is shown in Fig. 1a. The nanoparticulate nature of the material is also evident from the broadness of the peaks in its powder XRD pattern shown in Fig. 1b. The crystallite size in our samples is clearly smaller than that in somatoidal akaganéite samples prepared by slow hydrolysis of $\mathrm{FeCl}$
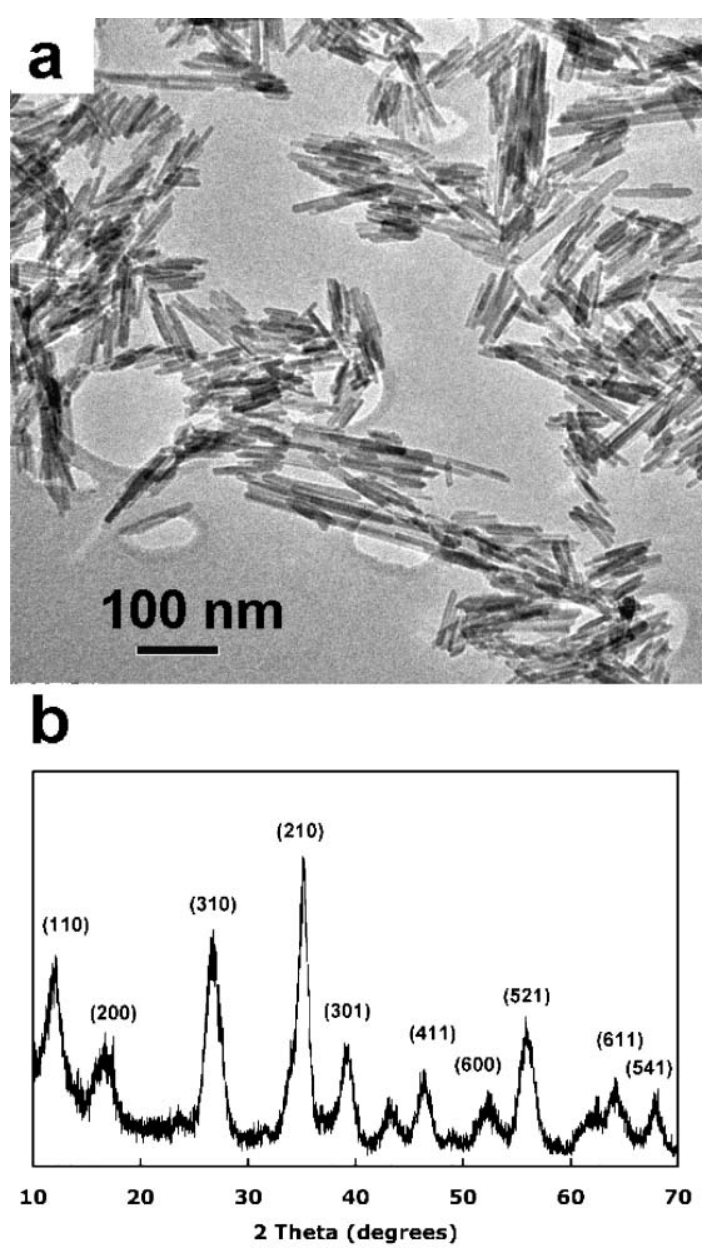

Fig. 1 a) TEM image of $\beta$-FeOOH particles showing the rod-like morphology. b) Powder XRD pattern of the sample shown in a). solutions, ${ }^{1}$ and the needles have a higher aspect ratio than those prepared by the method of Parfitt et al. ${ }^{9}$

Close examination of the akaganéite needles shown in Fig. 1(a) revealed that while most of the particles were solid rods, a small portion of the particles had the appearance of hollow tubes. The presence of tubes was observed in a number of samples which had been aged over slightly different times in the range 3-6 hours at $98{ }^{\circ} \mathrm{C}$. Visual examination of electron micrographs does not really allow a quantitative assessment of the proportion of tube-like particles in the samples, but our subjective estimate, based on our efforts to indentify tubes from a large number of micrographs, would be that less than $10 \%$ of the particles (by number) had the tube morphology. Some examples of the tube-like particles are presented in the micrographs shown in Fig. 2. Definitive identification of the tube-like morphology was not straightforward, due to the tendency of the particles to form close aggregates, but we have identified many instances where the contrast changes across the particle are consistent with the presence of hollow tubes and cannot be explained by the overlap of adjacent solid rods.

The clearest examples are those in which a single akaganéite tube extends across the edge of a hole in the carbon film. This is demonstrated in the image shown in Fig. 3, which shows an akaganéite tube in just such a situation. Lattice planes are visible running along the length of the tube, both on the outer edges, and within the "hollow" central portion, indicating that the tube is a single crystal of akaganéite.

Fig. $3 \mathrm{~b}$ shows the intensity profile across the tube, allowing us to measure the diameter of the hollow within the tube. Tube-like particles measured in this way were generally around $10-12 \mathrm{~nm}$ in diameter, with a central "hole" measuring approximately 3-4 $\mathrm{nm}$ in diameter. The solid rod-like particles however, were found to vary in diameter from as little as 3-4 $\mathrm{nm}$ up to about $15 \mathrm{~nm}$ in diameter. This observation suggests that the 3-4 nm rod-like particles may be a subunit in the formation of the larger rods and tube-like particles.

The particles did show some evidence of beam sensitivity, and prolonged exposure to the electron beam tended to cause the ends of the particles to become rounded and led to a pitted appearance of the edges of the particles. There was no case however, in which we saw reason to attribute the hollow appearance of the tubes to the effects of the beam. Galbraith et $a l^{8}$ demonstrated that electron beam irradiation is responsible for the appearance of mottling in cross-sections of somatoidal akaganéite, and this observation was used to refute earlier suggestions that these particles were composed of stacks of hollow tubes. In our samples however, images show clear evidence of a void extending along the length of the particles, which would seem to be far too uniform to be the result of degradation by the beam. Furthermore, we were unable to reproduce the appearance of the tube-like particles in attempts that involved deliberately exposing solid rod-like particles to the electron beam for a prolonged period.

\section{STEM imaging and nanodiffraction}

Bright-field STEM imaging of the akaganéite samples confirmed that they contained predominantly rod-like particles, but also a significant number of tubes with outside diameters 

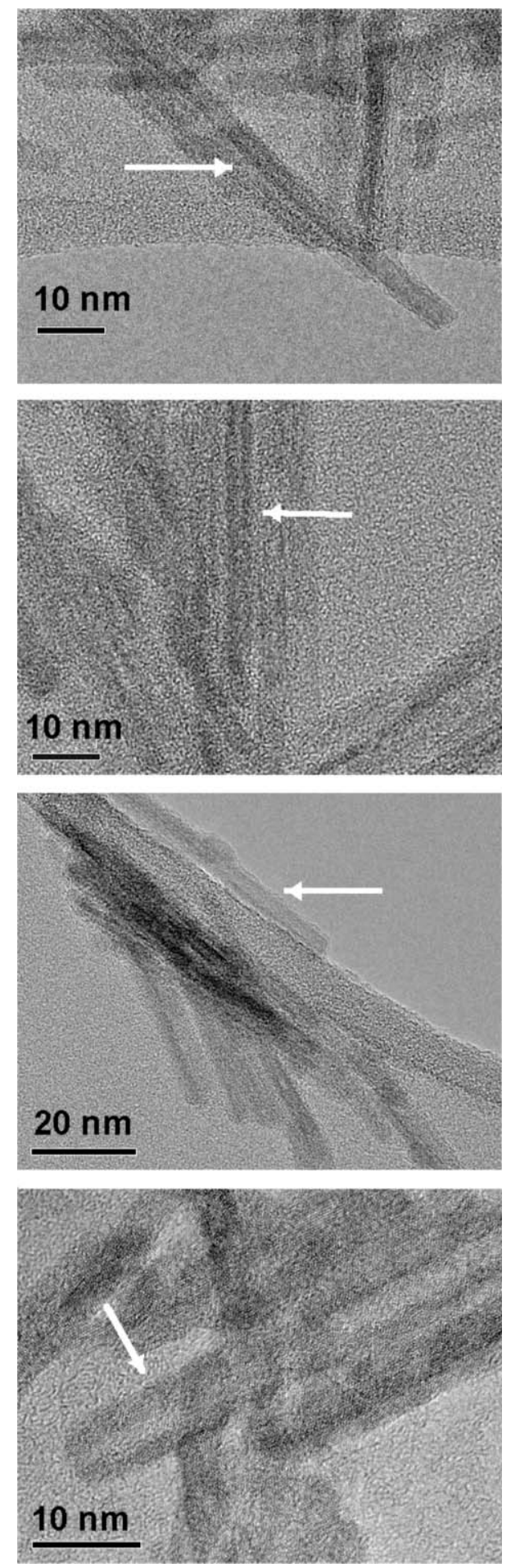

Fig. 2 TEM images of rod-like akaganéite particles with examples of the hollow tube morphology indicated by arrows.

ranging from about 10 to $15 \mathrm{~nm}$ and lengths about 10 times greater. Tube structures could be clearly seen in bright-field images, but the dark-field STEM images were more persuasive; the hollows in the tubes showed up clearly when the image intensities were dependent only on the projected atom density. An example of a dark-field STEM image obtained with a broad annular detector is shown in Fig. 4a, the intensity in this
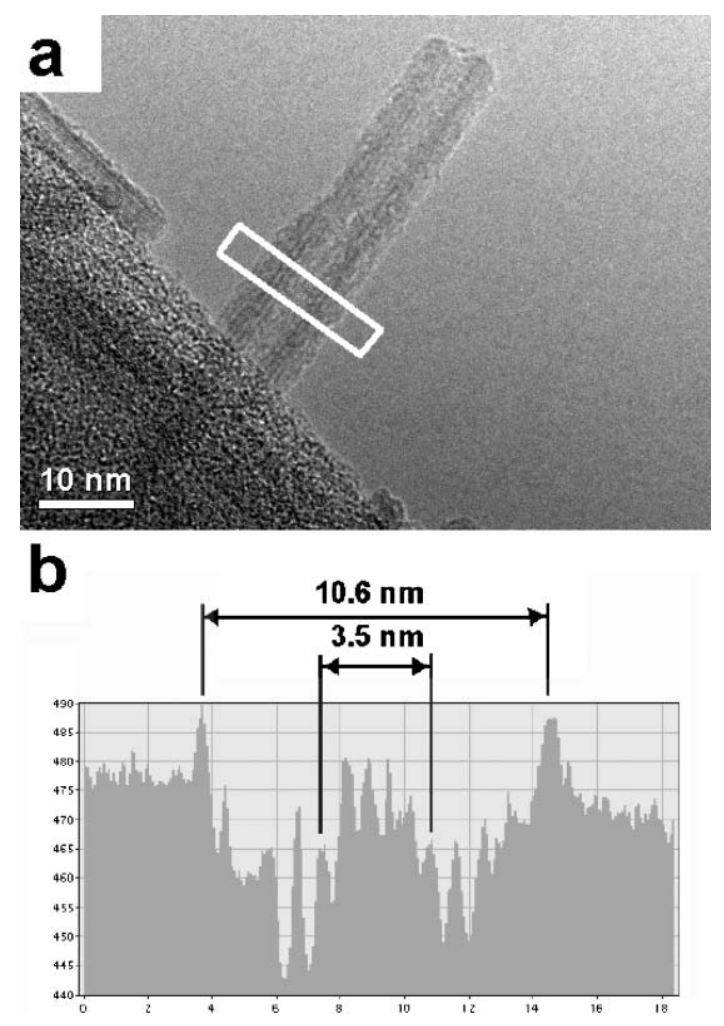

Fig. 3 a) TEM image of a hollow akaganéíte particle protruding over the edge of a hole in the carbon film. b) An intensity profile measured across the region of the white rectangle, showing the relative diameter of the particle and its hollow cavity.

image is approximately proportional to the number of atoms present, and particles of tube morphology (amongst a stack of parallel rods) can be seen in the centre of this image.

Some dark-field images of the samples were obtained with a thin annular detector, and thus with the electron beam located close to the annular detector, the image was given mostly by strong inner diffraction spots and showed the extent of individual single crystals. The image shown in Fig. $4 \mathrm{~b}$ is an example of this. For some of the tubes present, we found that a single crystal would extend over two sides of the tube image, but would usually not extend along the whole tube length - as is the case with the central particle in Fig. $4 \mathrm{~b}$.

Nanodiffraction patterns of the tube-like particles (about 30 were recorded) were generally less well developed than those obtained for the solid rods, which may be a result of radiation damage or the distortions associated with the tubular shape. Nonetheless, the patterns clearly demonstrated the akaganéite structure, with lines of spots having either the (110) spacing of $7.4 \AA$ or the (200) spacing of $5.2 \AA .{ }^{15}$ Several series of nanodiffraction patterns were recorded with the beam translated across tubes and it was found that the patterns given by the two sides and the middle of a tube were much the same, with minor differences representing only a few degrees difference in orientation. Hence we conclude that the tubes are actually single crystals, somewhat distorted by having the hole in the middle, but reasonably coherent nevertheless. The single crystals do not usually extend all the way along a tube, but there may be changes in the structure several times along a 

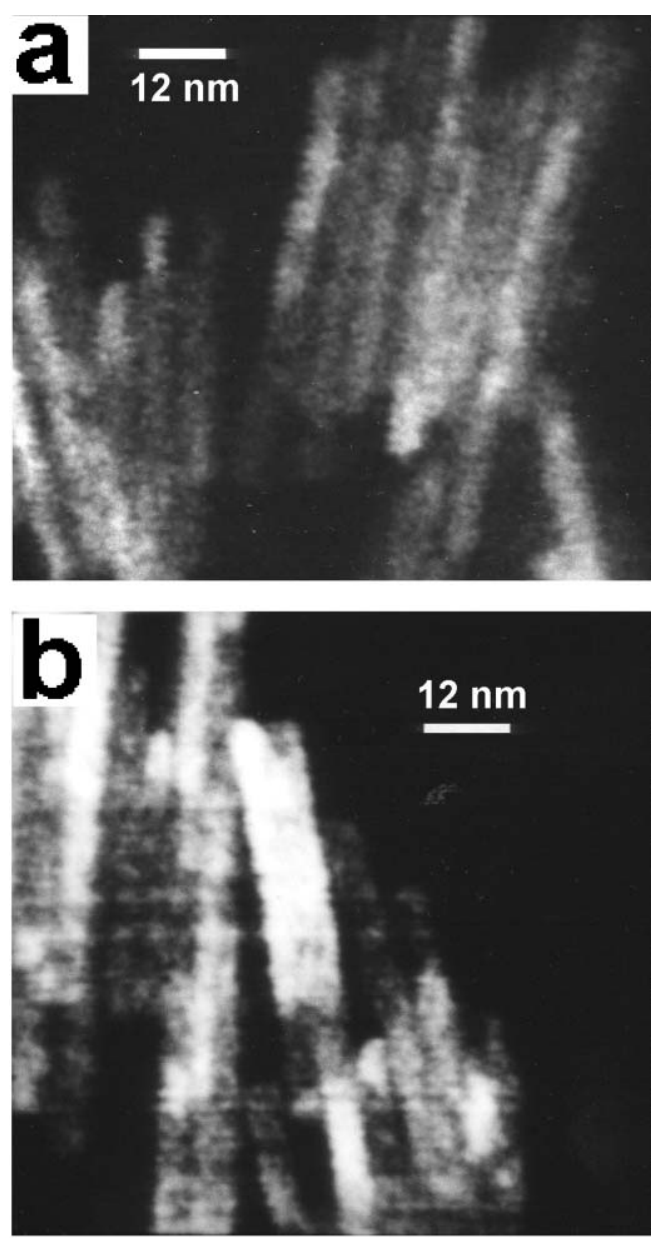

Fig. 4 Dark-field STEM images of akaganéite samples containing particles with the tube morphology. a) Image obtained with annular detector close to the electron beam. b) Image obtained with the detecor far from the electron beam.

tube's length. It was however, difficult to trace the changes in crystal structure along a tube, because most of the tubes occurred in overlapping, near-parallel clumps on the TEM grids. By greatly defocusing the objective lens so that each diffraction spot displayed a small image of the crystallite, we were able to determine the orientation of the crystals relative to the tube and confirm that the $c$-axis of the tetragonal cell, and hence the orientation of channels in the akaganéite structure, is parallel to the tube axis.

\section{Formation of tubes}

As mentioned earlier, the dimensions of the tubes, along with the observation that the hollows of the tube-like particles are always about $1 / 3$ of the tube diameter, seem to suggest that the tubes are made up of rod-like subunits of $c a .3-4 \mathrm{~nm}$ in diameter. On the basis of this observation, some possible mechanisms for the formation of the tubes can be inferred.

One possibility is that $3-4 \mathrm{~nm}$ wide rods form initially in the condensed gel, growing rapidly in the $c$-direction, but with little or no growth along other crystal axes. These rods may then assemble into larger particles by alignment along their long axes to produce rods or tubes of greater diameter. Another possibility is that primary nuclei of 3-4 nm dimensions form and then grow rapidly in the $c$-direction, followed by secondary nucleation occurring at sites on the edges of these rods. Thus we would have a system in which particles could lengthen by rapid growth in the $c$-direction, but could only become wider by formation of a new 3-4 nm nucleus on their long edge. Rod-like particles might result most of the time, but tubes would be formed when growth from these new nuclei happened to completely enclose a void. The second of these two possible mechanisms seems to be more consistent with the types of particles we have observed in our samples, especially in light of the fact that most tubes were found to have single coherent crystals extending across the width of a tube particle, while single crystals were only rarely found to extend along the whole length of a tube.

The TEM image shown in Fig. 5 provides some support for this proposed mechanism, as it shows a cross-sectional view of a number of rods of different diameters. The smallest of these do appear to be about $3 \mathrm{~nm}$ in diameter, and there is a particle to the left of the image that has an L-shaped cross-section. This is one of the forms we would expect to see if the rods and tubes were indeed growing by assembly of smaller subunits, or by a contact nucleation process as just described. The crosssectional view shown in Fig. 5 resulted from the fortuitous alignment of a few particles on the edge of a hole in the carbon film in an orientation perpendicular to the TEM grid. In spite of numerous attempts at imaging resin-embedded crosssections of our akaganéite samples, we have so-far been unable to obtain high-quality cross-sectional images of the tube morphology.

Why a 3-4 nm wide nucleus should be the principal subunit to form in this system is not clear. The akaganéite unit cell length is $1.5 \mathrm{~nm}$ so the subunit dimensions correspond to approximately 2 or 3 unit cells. This is very similar in size to the subunits proposed by Gallagher ${ }^{4}$ for somatoidal akaganéite particles, with the important difference that Gallagher indicated that these subunits were themselves hollow tubes, while we propose that the subunits have no void within them,

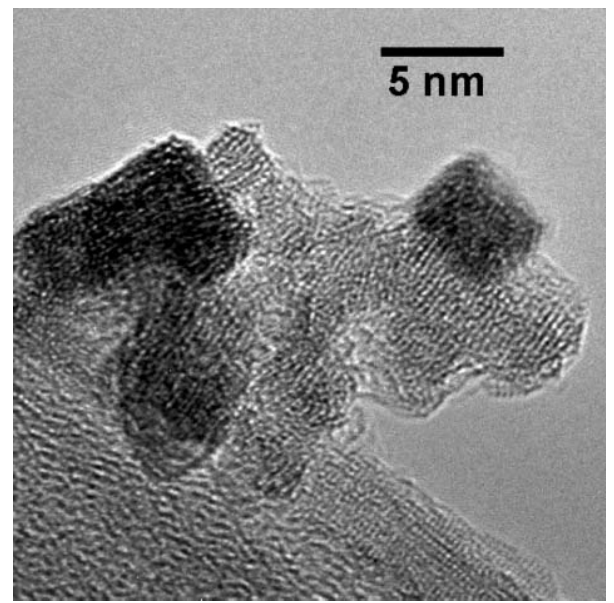

Fig. 5 TEM image showing a group of rod-like akaganéite particles viewed end-on as they sit on the edge of a hole in the carbon film. The particle on the left of this image has an L-shaped cross-section. 
but are able to grow in such a way as to enclose a void, thus forming a square-sided hollow tube of larger dimensions.

\section{Conclusions}

$\beta-\mathrm{FeOOH}$ samples prepared by quenching of a condensed iron(III) hydroxide gel were found to contain a mixture of rodlike nanoparticles along with nanotubes about $10-15 \mathrm{~nm}$ in diameter and with a hollow centre that was typically about $1 / 3$ of the particle diameter. The discovery of such tubes is remarkable because a very similar morphology was proposed as a building-block for somatoidal $\beta-\mathrm{FeOOH}$ by other researchers in the $1960 \mathrm{~s}$, but subsequently discounted as an artefact resulting from beam damage in the electron microscope. The observations described in this study show that nanotubes of $\beta-\mathrm{FeOOH}$ can form nonetheless, although we see no reason to discount the findings of earlier studies, which focused only on the morphology of somatoidal akaganéite particles. The reaction conditions we have employed, precipitating the akaganéite phase from a concentrated ferrihydrite gel at $98{ }^{\circ} \mathrm{C}$, provide for much more rapid kinetics than the conditions usually employed in the synthesis of somatoidal akaganéite, so it is possible that the tube like particles are essentially metastable and would therefore be less likely to form in systems that were allowed a longer reaction time. Identification of the factors controlling tube formation and ultimately, directed synthesis of akaganéite nanotubes, are important goals for future research, as particles of this morphology may have interesting potential applications in the development of nanoscale devices.

\section{Acknowledgements}

This work was partially supported by funds from the Australian Research Council's Discovery Grant Scheme through Discovery Grant DP0451535.

\section{References}

1 R. M. Cornell and U. Schwertmann, The Iron Oxides, Wiley-VCH, Weinheim, 2003.

2 J. H. L. Watson, R. R. Cardell and W. Heller, J. Phys. Chem., 1962, 66, 1757.

3 K. J. Gallagher and D. N. Phillips, Chimia, 1969, 23, 465.

4 K. J. Gallagher, Nature (London), 1970, 226, 1225.

5 J. M. González-Calbet, M. A. Alario-Franco and M. GayosoAndrade, J. Inorg. Nucl. Chem., 1981, 43, 257.

6 H. Naono, R. Fujieara, H. Sugioka, K. Sumiya and H. Yanazawa, J. Colloid Interface Sci., 1982, 87, 317.

7 E. Paterson and J. M. Tait, Clay Miner., 1977, 12, 345.

8 S. T. Galbraith, T. Baird and J. R. Fryer, Acta Crystallogr., Sect. A, 1979, 35, 197.

9 R. L. Parfitt, R. J. Atkinson and S. R. C. St., Soil Sci. Soc. Am. Proc., 1975, 39, 837.

10 T. Sugimoto, Monodispersed Particles, Elsevier Science, Amsterdam, 2001.

11 J. M. Cowley and J. C. H. Spence, Electron Nanodiffraction, in Handbook of Nanostructured Materials and Nanotechnology, Volume 2: Spectroscopy and Theory, ed. H. S. Nalwa, Academic Press, New York, 2000.

12 J. M. Cowley, D. E. Janney, R. C. Gerkin and P. R. Buseck, J. Struct. Biol., 2000, 131, 210.

13 D. E. Janney, J. M. Cowley and P. R. Buseck, Clays Clay Miner., 2000, 48, 111.

14 M. Loan, R. D. Hart and J. M. Cowley, Am. Mineral., 2004, 89, 1735.

15 J. E. Post and V. F. Buchwald, Am. Mineral., 1991, 76, 272. 